Revue d'histoire de l'Amérique française

\#A REVUE D.HISTOIRE DE L'AMÉRIQUE FRANÇAISE

\title{
Le cinquième anniversaire de la Société Généalogique canadienne-française
}

\section{Conrad-M. Morin}

Volume 2, numéro 4, mars 1949

URI : https://id.erudit.org/iderudit/801503ar

DOI : https://doi.org/10.7202/801503ar

Aller au sommaire du numéro

Éditeur(s)

Institut d'histoire de l'Amérique française

ISSN

0035-2357 (imprimé)

1492-1383 (numérique)

Découvrir la revue

Citer cet article

Morin, C.-M. (1949). Le cinquième anniversaire de la Société Généalogique canadienne-française. Revue d'histoire de l'Amérique française, 2(4), 576-580. https://doi.org/10.7202/801503ar d'utilisation que vous pouvez consulter en ligne.

https://apropos.erudit.org/fr/usagers/politique-dutilisation/ 


\section{LE CINQUIEME ANNIVERSAIRE DE LA SOCIẼTÉ GÉNÉALOGIQUE CANADIENNE-FRANÇAISE}

Notre Revue ne saurait passer sous silence le cinquième anniversaire de la Société Généalogique Canadienné-Française. Il y a, en effet, entre la généalogie et l'histoire trop de liens étroits, trop d'utilité réciproque pour ne pas nous réjouir fort de la vitalité de cette sympatique association de généalogistes et de travailleurs d'archives.

Elle est l'œuvre d'un expert en généalogie canadienne, le meilleur que je sache, le R.P. Archange Godbout, O.F.M., membre de la Société Historique de Montréal et auteur du très utile ouvrage de documentation qu'est Origine des familles canadiennes-françaises (Lille, 1925). Fréquemment consulté en la matière, par ses compatriotes et autres, le R.P.'se rendit vite compte de deux faits: le nombre croissant de généalogistes et la similitude des difficultés entravant leurs. recherches. Il crut alors que bien des obstacles seraient levés si les amateurs de généalogie se groupaient en une association qui leur permit de se renseigner mutuellement, " soit en faisant bénéficier les autres membres des notes qu'ils possédaient déjà, soit en faisant des recherches dans leur localité ").

$\mathrm{Au}$ printemps de 1943, il adressa donc une circulaire approprice à toutes les personnes qu'il croyait intéressées aux études généalogiques. Le résultat favorable de l'enquête permit de faire le pas décisif: le 3 septembre 1943, un groupe de connaisseurs, constitué en assemblee délibérante à 2080 ouest, rue Dorchester, Montréal, en confiait la présidence au fondateur et lui adjoignait M. J.-Alfred Perrault, comme trésorier, et M. Casimir Hébert, comme secrétaire, puis adoptait le projet de constitution soumis. La Société Généalogique Canadienne-Française était née. Le 15 septembre, la première assemblée plénière de la Société - qui fut un "véritable congrès" à cause des participants venus d'un peu partout - ratifiait le choix déjà fait 
de la constitution et l'exécutíf, tout en augmentant celui-ci, pour se conformer à la constitution, d'un vice-président, d'un bibliothécaire et de deux conseillers. Enfin, le 24 novembre, des lettres patentes obtenues du Gouvernement de la Province de Québec par l'entremise de l'hon. Oscar Drouin, ministre des Affaires municipales, de l'Industrie et du Commerce, conféraient l'existence légale à la Société. Désormais, elle pouvait donc se livrer en toute sécurité à la réalisation de son noble idéal: " répandre les connaissances généalogiques et l'histoire des familles, par gratitude envers les aieux et pour maintenir les traditions ancestrales )" (art. 2).

Toutefois, pour y être reçu comme membre, il ne suffit pas de payer la cotisation annuelle (deux dollars). La Société, en effet, ne peut recevoir n'importe quel intéressé, puisqu'elle est une association " de personnes qui ont fait quelques travaux généalogiques et consentent a en faire profiter gratuitement les autres membres de la Société ") (art. 3). Et cette entr'aide gratuite n'est pas un vain mot: " on a cré un dépôt d'archives et de documents généalogiques à la Bibliothèque municipale " de Montréal, où "les membres sont invités à verser leurs ou vrages, archives, copies d'archives " (art. 11). Ils a'en perdent pas toutefois la propriété, puisque les usagers (c'est-à-dire les seuls membres de la Société) ne sont (" autorisés à les photographier ou à les copier en entier que sur autorisation expresse des possesseurs " (art. 11). Cette mise en commun de la documentation des membres n'est pas la seule utilité du dépôt d'archives de la Société. Il doit comprendre tout ce que celle-ci aura pu se procurer, même moyennant achat, concernant " l'histoire de nos familles: livres, brochures, extraits de revues ou de journaux, cartes, photographies, actes notarís, livres de raison, textes de toute nature en origiaaux ou en copies".

En vertu de sa charte comme de sa constitution, la Socióté a son siège social a Montr6al, actuellement localisé à la Bibliothèque Municipale, où elle tient régulièrement ses assemblés, le deuxième mercredi de chaque mois. Mais elle a prévu l'établissement, " dans d'autres villes, de filiales régies par la même constitution w (art. 1). Elle en compte cinq actuellement: Ottawa, Québec, Trois-Rivières, SaintHyacinthe et La Sarre. Cette heureuse stipulation semble avoir contribu6 fort a son rapide accroissement: le 3 septembre dernier, jour ou elle accomplissait le cinquième anniversaire de son existence, elle avait largement dépaesé les cinq cents membres. Pour donner des chiffres exacts: 
555 membres inscrits, dont 22 décédés. Des 533 vivants, 371 étaient laĩques des deux sexes ( 85 femmes), 76 religieux et 86 prêtres séculiers. On en comptait de diverses régions de la Province de Québec (180 de l'Ile de Montréal, 240 d'ailleurs) et de tout le Canada (55) ainsi que des Etats-Unis (45), d'Europe même (13). Aussi est-il tout normal que la Société ait voulu souligner sa cinquième année de vie par la collation de diplômes de " membre émérite " à quelques-uns de ses chercheurs les plus remarquables: M. E.-Z. Massicotte et le T.R.P. Anthime Desnoyers, O.M.I., à Montréal (14 avril); M. le chanoine Pierre-A. St-Pierre, à Saint-Hyacinthe (7 avril); M. Pierre-Georges Roy et le Rév. Frère Sigismond, E.C., à Québec (14 avril); R.P. Jean-Louis Bergevin, O.M.I., Dom Adélard Bouvier, O.S.B., et le Rév. Frère Eloi-Gérard, F.M., à Montréal (8 septembre). Il eût été étonnant que la Société n'ait pas songé à marquer de la même façon l'œuvre si considérable et si utile de son fondateur, le R.P. Archange Godbout, O.F.M. Elle le fit également le 8 septembre, à la Bibliothèque municipale de Montréal.

Un autre article constitutionnel mérite notre attention: "Les publications de la Société seront adressées gratuitement, mais aux membres seulement qui auront payé leur cotisation " (art. 10). Là encore ce n'étaient pas de vains mots. Quatre mois à peine après la naissance de la Société, en janvier 1944, paraissait le premier numéro de son bulletin semestriel: Mémoires de la Société Généalogique Canadienne-Française, in-octavo $(17,5 \times 26,0 \mathrm{~cm})$ de 72 pages. Ce périodique, par conséquent, vient donc d'accomplir, lui aussi, sa cinquième année d'existence. Il comporte deux livraisons par année, soit un volume par deux ans, de sorte que la livraison de juin 1948 - la dernière de la cinquième année - forme le numéro 2 du vol. III. Son contenu se répartit ordinairement en quatre sections: I) La Société généalogique (rapport du secrétaire, filiales, etc.); II) Généalogie et petite histoire (articles généraux d'utilité généalogique); III) Etudes généalogiques proprement dites; IV) Chronique généalogique; V) Entre nous (liste des membres mise à date; liste des archives privées mises a la disposition des membres par ordre alphabétique de noms de familles et de lieux, avec indication de leurs possesseurs respectifs; enfin, questions et réponses). A la fin de chaque volume, un très commode index alphabétique permet au lecteur de retrouver facilement l'objet de ses recherches. Comme chaque membre porte un numéro 
distinctif, qui lui est donné dès son admission dans la Société et qui ne change jamais, il est ainsi possible d'indiquer les possesseurs des archives privées et les auteurs des questions et des réponses sans prendre trop d'espace dans le texte.

Inutile de dire que ces Mémoires périodiques sont très appréciés des membres, tant pour l'intérêt que pour l'utilité qu'ils comportent. Aussi a-t-on songé à les publier trimestriellement. En attendant que ce projet puisse se réaliser, on a résolu le problème par une fort heureuse initiative: Le Mois généalogique, feuille mensuelle de quatre pages, de même format que les Mémoires, qui sert aux membres toute nouvelle de nature à les intéresser. Ce nouvel organe, qui paraît régulièrement depuis janvier 1948, a eu pour bon effet non seulement de tenir sans cesse les associés au courant des activités de leur Société, mais aussi de créer entre eux un lien " familial " plus étroit, plus dynamique. Une preuve écla tante de la chaleureuse réception que l'on fait chaque fois au Mois, c'est que chacun de ses numéros (au cout de quinze dollars) est payé par des bienfaiteurs.

La Société Généalogique - on s'en est sans doute rendu compteprésente donc beaucoup d'affinité avec l'Institut d'Histoire de l'Amérique Française. Dans l'une comme dans l'autre, c'est un travail d'équipe et ce travail a pour objet le fait français d'Amérique. Mais, alors que l'Institut traite celui-ci dans son évolution générale, qu'il en scrute les détails en fonction du tout, la Société Généalogique, au contraire, pousse ses investigations dans un champ limité: la famille canadienne-française. Par conséquent, ce que l'Institut est à l'histoire générale de l'Amérique française (spécialement du Canada), ce que nos sociétés historiques sont à l'histoire régionale du pays, la Société Généalogique, elle, l'est à l'histoire domestique canadienne. Il est dès lors facile de s'imaginer l'interdépendance de ces trois catégories de chercheurs: l'Institut a besoin de la Société généalogique et de nos sociétés historiques pour mieux trouver toute la matière a synthétiser, tandis que celles-ci ont besoin de celui-là pour mieux saisir la valeur et la portée des détails du fait français chez nous. Aussi rien d'étonnant que le fondateur-président et d'autres membres de la Société Généalogique comme la plupart de nos sociétés historiques fassent partie de l'Institut d'Histoire de l'Amérique Française; d'autre part, rien d'étonnant aussi que le fondateur-président et bien 
d'autres membres de l'Institut se trouvent dans les cadres des susdites sociétés.

D'ailleurs, abstraction faite de toutes ces affinités et autres, la naissance et la croissance de la Société Généalogique, comme celles de nos sociétés historiques et de l'Institut, ne sont-elles pas un éclatant témoignage de la vitalité profonde du fait français? A titre documentaire, notre Revue devait done saluer de la sorte les cinq ans de vie de la Société Généalogique, se réjouir de son accroissement continu et de son activité incessante. Nos lecteurs nous sauront donc gré, sans doute, de lui souhaiter, en terminant, bien d'autres quinquennats de vie et de succès.

Université de Montréal

Conrad-M. Monin, O.F.M.

Professeur d'histoire d la Faculte des Lettres 